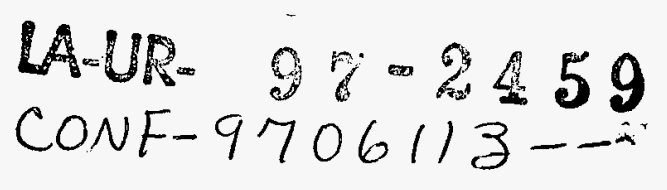

\title{
EJECTA EXPERIMENTS AT THE PEGASUS PULSED POWER FACILITY
}

\author{
D.S. Sorenson, B. Carpenter, N.S.P. King, D. Morgan, A. W. Obst, D. Platts, P. Rodriguez, J. \\ Roberts, M. Stelts, J. Stokes, T. Taylor, L. Veeser. \\ Los Alamos National Laboratory, Los Alamos, NM, 87544 \\ B. Frogget, B. Malone, T. Tunnell, D. Westley \\ Bechtel Nevada, Los Alamos, NM 87544 \\ R. Flurer \\ Bechtel Nevada, Las Vegas, NV, 89125

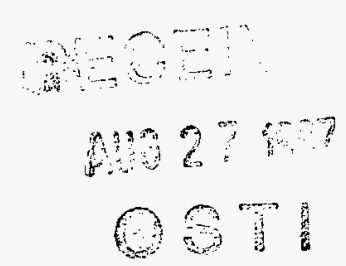

\begin{abstract}
When a shock wave interacts at the surface of a metal target, target material can be emitted from the surface called ejecta. The mass, size, shape and velocity of ejecta varies depending on the initial shock conditions, and target material properties. In order to understand this phenomena, diagnostics have been developed and implemented at the Pegasus Pulsed Power facility located at Los Alamos National Laboratory. The facility provides both radial and axial access for making measurements. There exist optical, laser, and $\mathrm{x}$-ray paths for performing measurements on the target assembly located near the center of the machine. The facility can provide many megaamps of current which is transported to a $5.0 \mathrm{~cm}$ diameter, $2.0 \mathrm{~cm}$ high aluminum cylinder. The current and associated magnetic field set up forces which implode the aluminum cylinder radially inward. As the aluminum cylinder reaches the appropriate velocity it impacts a target cylinder. Due to this impact, a shock wave is set up in the target and eventually interacts at the inner surface of the target cylinder where ejecta are produced. A $1.5 \mathrm{~cm}$ diameter collimator cylinder located inside the target cylinder is used to control the number of ejecta particles that arrive at the center region where ejecta measurements are made. Diagnostics have been developed including in-line Fraunhofer holography and visible shadowgraphy. Details of the these diagnostics will be described.
\end{abstract}

\section{Introduction}

Metals under shock loaded conditions can lead to complex phenomena depending on varying properties of the material and initial shock conditions. In particular, if a metal is shocked debris can be emitted from the surface as the shock interacts at the surface. In order to characterize the debris two diagnostics have been developed and are being applied on experiments conduced at the Pegasus Pulsed Power Facility. The first diagnostic developed is holography which is primarily used to provide three-dimensional imaging with the capability of measuring particles down to 2 microns is size. The second diagnostic is visible shadowgraphy which provides spatially resolved images for typically twelve different times. In this report the experimental setup will be described and the diagnostics discussed. The goal of these experiments is to characterize ejecta for various target and shock conditions. Many experiments similar to these have been performed at other facilities ${ }^{1,2,3,4}$.
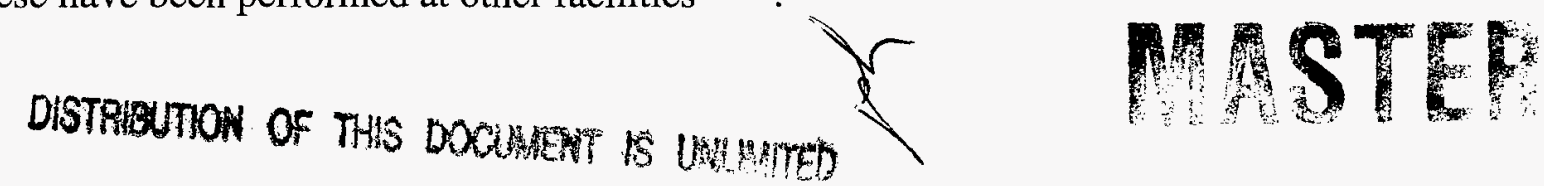


\section{DISCLAIMER}

This report was prepared as an account of work sponsored by an agency of the United States Government. Neither the United States Government nor any agency thereof, nor any of their employees, make any warranty, express or implied, or ascumes any legal liability or responsibility for the accuracy, completeness, or usefulness of any information, apparatus, product, or process disclosed, or represents that its use would not infringe privately owned rights. Reference herein to any specific commercial product, process, or service by trade name, trademark, manufacturer, or otherwise does not necessarily constitute or imply its endorsement, recommendation, or favoring by the United States Government or any agency thereof. The views and opinions of authors expressed herein do not necessarily state or reflect those of the United States Government or any agency thereof. 


\section{Ejecta Formation}

Shock strengths of many hundreds of kilobars can be obtained in $\mathrm{Al}$ and $\mathrm{Sn}$ targets at the Pegasus Pulsed Power Facility. When the shock wave interacts at the target vacuum interface ejecta can be emitted from the surface. Fig. 1 illustrates a target in which defects in the material, grain size, and surface finish can all contribute to how the material may break up as the shock

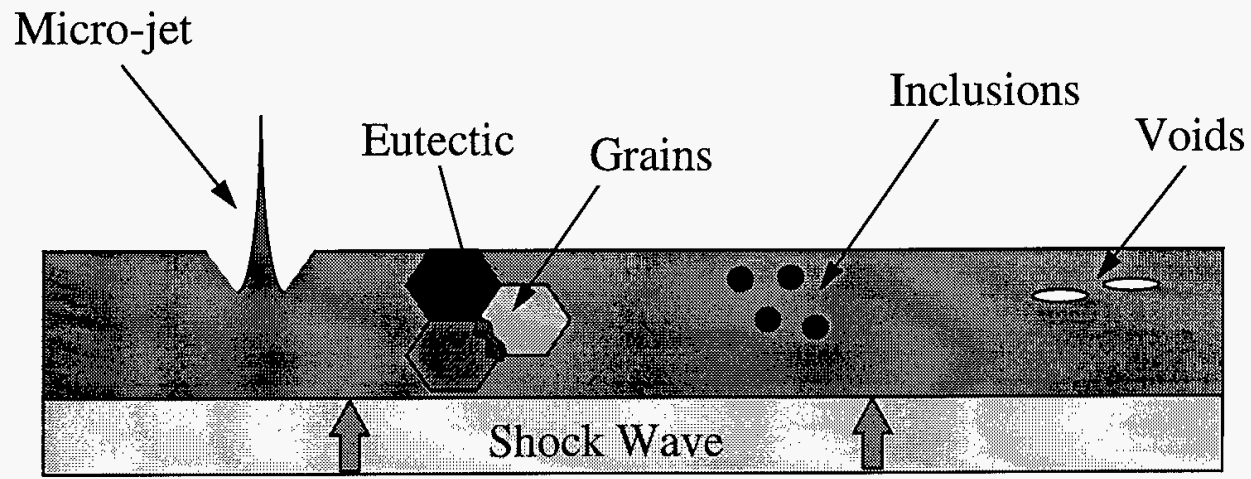

Figure 1: Illustration of possible ejecta formation processes. The shock wave is shown coming from the bottom. The shock wave can interact in the material causing the material to break up and be ejected out ahead of the material. wave interacts in the material and at the surface. In addition, the initial shock conditions contribute strongly in determining the amount and velocity of the ejected material. Furthermore, if the shock wave is strong enough the material can melt at which point the material has to treated as a liquid. Experiments are designed so that material and shock wave properties are well characterized. The corresponding experimental measurements can then be correlated with those properties that were changed. As an example,

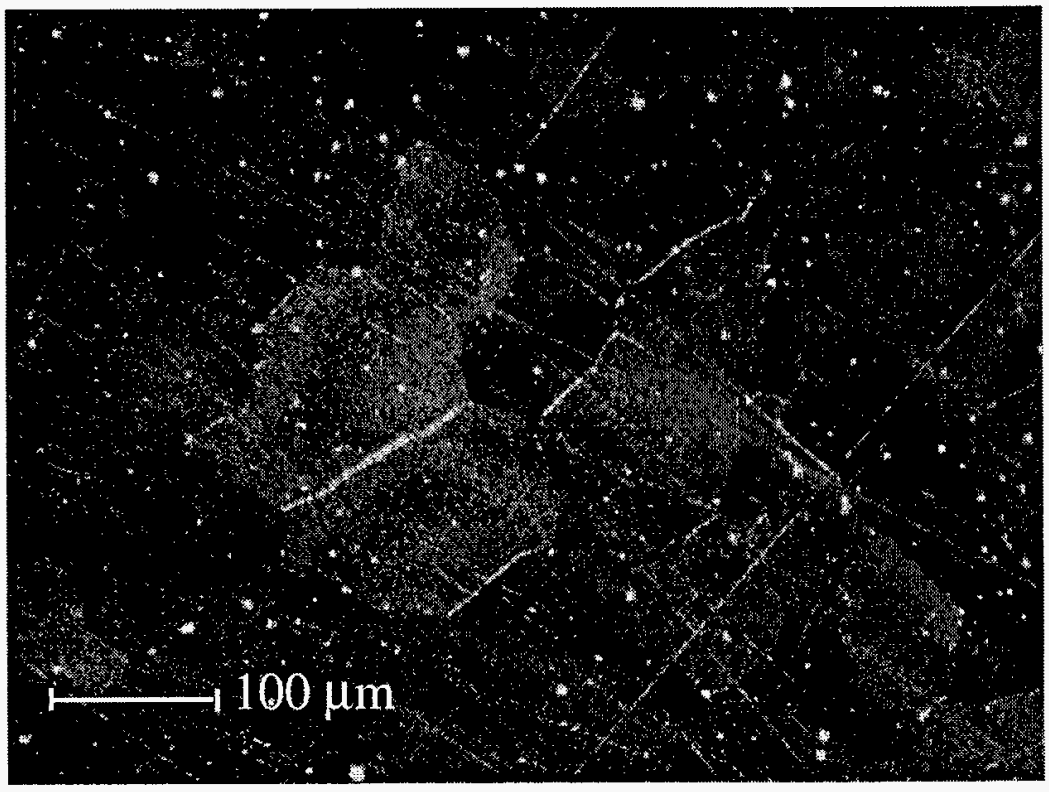

Figure 2: Photomicrograph of Sn. The target has been etched to bring out the grain boundaries.

will be described. two material properties that may contribute to ejecta are the grain size of the material and the inclusions. Fig. 2 shows a photomicrograph of $\mathrm{Sn}$. The figure shows that the grain sizes on the average are 70 microns in size. These grains could break apart as the shock wave passes through the material. Inclusions may also offer a means for ejecta production. Depending on the amount and type of inclusions large density discontinuities can be present in the material which could break apart as the shock wave passes through the material. In the rest of the report the Pegasus Facility and associated ejecta diagnostics 


\section{Ejecta Measurements at the Pegasus Pulsed Power Facility}

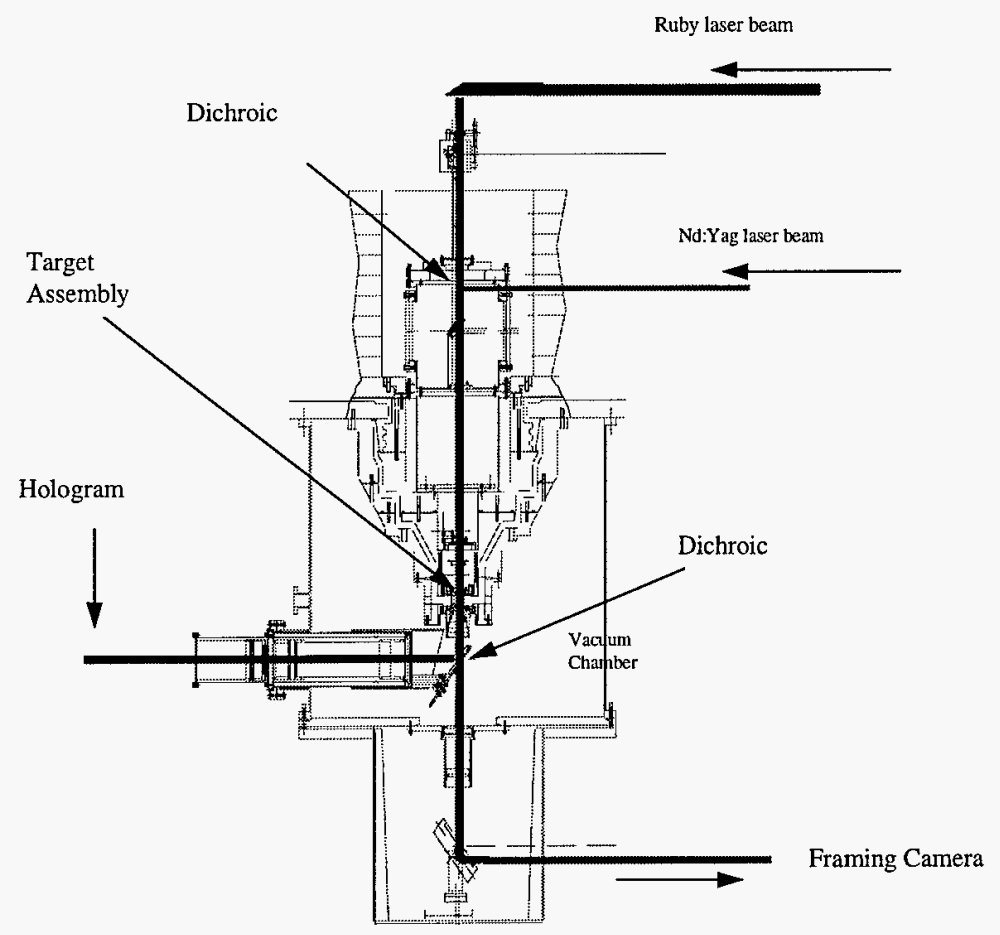

Figure 4: The ejecta experimental configuration at the Pegasus Pulsed Power Facility. Laser beams for the ejecta measurements are shown.

the liner which is the target. A 400-micron thick Sn target is used and when the liner $\mathrm{Al}$ cylinder impacts the Sn shock waves of many hundreds of kilobars can be achieved depending on the experimental requirements. Finally, another cylinder is used which is simply a Ta cylinder with various cutouts. The density of the ejecta formed from the collision of the liner with the target is reduced to an acceptable level for the holography measurements. Various diagnostics are used to characterize the performance of the Pegasus Pulsed Power machine. These include primarily current measurements which are done using B-dot probes and Faraday rotation technique ${ }^{5,6}$. In addition, one of the probes has proved useful in providing a trigger for the holography experiment. The initial conditions for the ejecta experiment start once the shock wave is set up in the target cylinder. This time
The Pegasus Pulsed Power (PPP) Facility provides current necessary to implode an aluminum cylinder to velocities of many $\mathrm{mm} / \mu \mathrm{sec}$. The experimental configuration is shown in Fig. 3. The figure shows the power flow channel which transports the electrical current to the heart of the machine where the physics experiments are performed. The experiments are conducted where both radial and axial access is available for making measurements. Fig. 4 shows a more detailed view of the target assembly area. For this particular experiment a 400 micron thick Al cylinder $2.0 \mathrm{~cm}$ high and 4.8 $\mathrm{cm}$ in diameter accepts the current and is driven cylindrically inward. This cylinder is called the liner driver. Another cylinder measuring $3.0 \mathrm{~cm}$ in diameter is shown inside

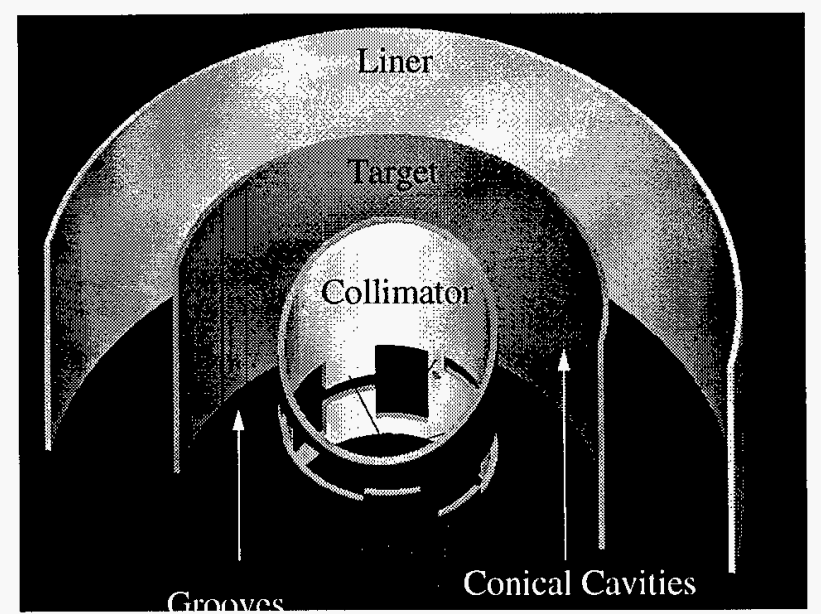

Figure 3: Target assembly. The liner driver is what the Pegasus facility drives with current. The target is where the shock wave is initiated after the liner impacts the target. The collimator is used to control the density of ejecta in the center region where the measurements are made. 
occurs about 9 microseconds after current has started to flow through the liner and the ejecta diagnostics are triggered about 3 microseconds later. If the ejecta diagnostics are triggered from the start of the current flow an error of $+/-100$ ns can be expected due to uncertainties in the charging of the Pegasus capacitors. To improve the timing uncertainty, the B-dot signal is put through a high pass filter, which passes the induced signal that occurs when the liner impacts the target. This signal is shown in Fig. 5 as the long dash curve and occurs at about 9 microseconds.

The figure also shows two other digitizer records in which the zero of the time scale corresponds to the start of current flow. The solid curve is a line.

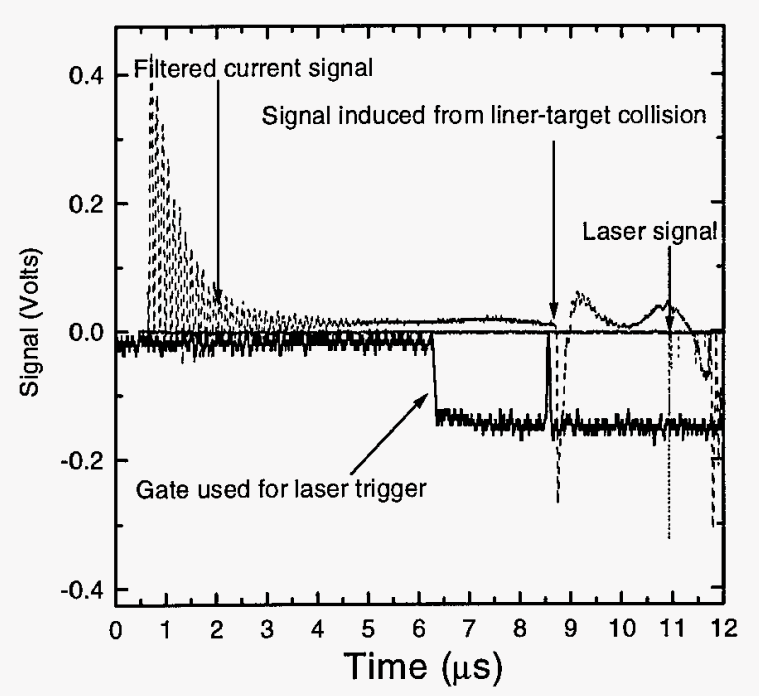

gate that is used in coincidence with the laser trigger so as to prevent a pretrigger which may occur at earlier times. Also shown in the figure is the actual laser monitor signal which records the laser light as it passes through the hologram. Another diagnostic that is used to measure the dynamics of the target assembly are three radial $\mathrm{x}$-ray units ${ }^{7}$ used in conjunction with film to provide images of the cylindrical target assembly as it implodes. Fig. 5 shows a static and one of the three dynamic images obtained on an experiment. The outer liner, target cylinder, and collimator are labeled in the top figure. The Ta collimator completely absorbs the x-rays and so is completely black. The lower figure shows the experimental image. The target is seen to have moved inward from its original position, and some debris is left behind which is probably the $\mathrm{Al}$ liner driver. In addition to the X-ray imaging, visible imaging is used to measure the liner before

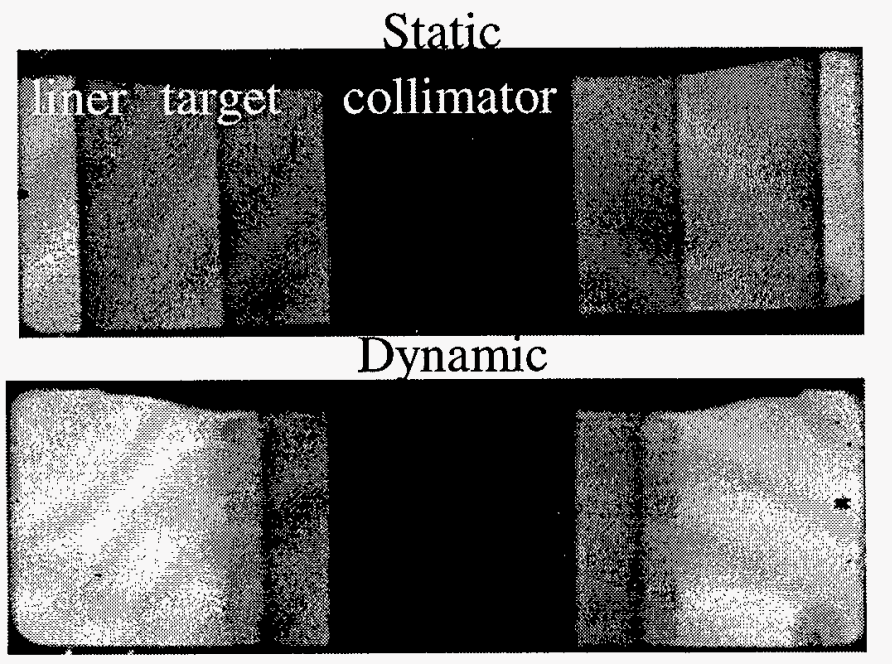

Figure 5: $\mathrm{X}$-ray image of target assembly. Image is viewing from the directions oriented radially out from the axis of the cylinders. the liner has impacted the target. With the $\mathrm{x}$-ray imaging, visible imaging, and current probes the target assembly dynamics can be accurately measured.

\section{Ejecta Diagnostics}

Two primary diagnostics are used to measure the ejecta emitted from the shocked surface. These are: in-line Fraunhofer Holography, and visible shadowgraphy. The in-line Fraunhofer holography has been described previously ${ }^{8,9,10,4}$ Fig. 2 shows how the laser beams for both of these systems 
traverse through the Pegasus vacuum chamber and the target assembly. Recently, a new optical relay system was designed and built to provide $1000 \mathrm{lp} / \mathrm{mm}$ resolution for an image plane $15 \mathrm{~mm}$ in diameter. This lens is used to relay an interference pattern produced close to the ejecta to a distance $93 \mathrm{~cm}$ from this plane. This layout of the lens is shown in Fig. 7. After the interference pattern of the ejecta is recorded onto the hologram the data is reconstructed optically and put into digital form. This process is described in Ref. 8.

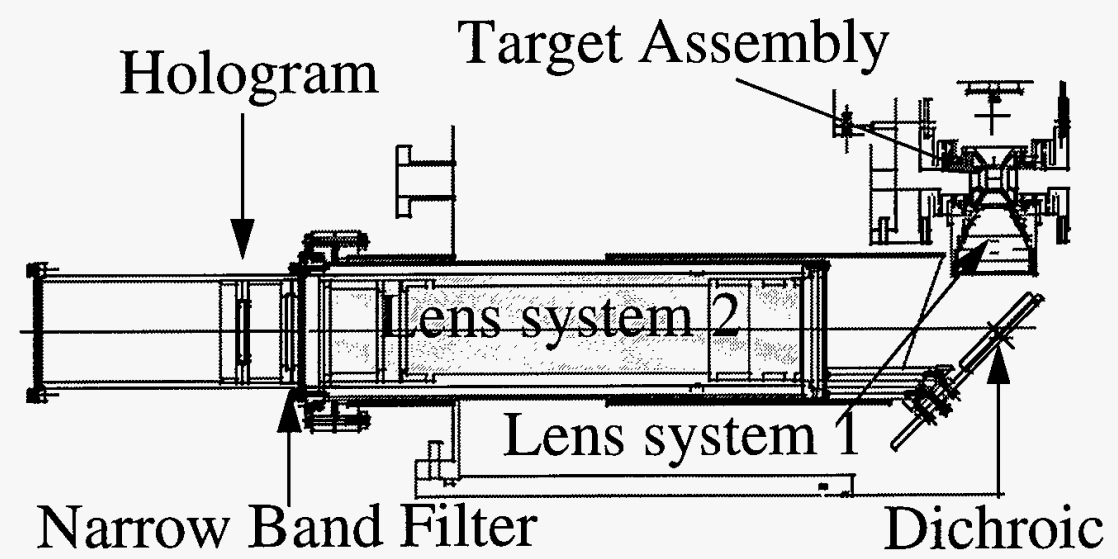

Figure 7: Lens system used for relaying the interference pattern. The hologram is located $93 \mathrm{~cm}$ from the image located in the target assembly. Dichroic shown is used to separate the Nd:Yag laser beam and the Ruby laser beam. The individual lens elements are not shown.

The shadowgraphy makes use of an 8 joule long pulsed ruby laser $(500 \mu \mathrm{s}$ FWHM). The ruby laser wavelength is $694 \mathrm{~nm}$ and the holography laser uses a wavelength of $532 \mathrm{~nm}$. This allows dichroic mirrors to be used shown in Fig. 3 and Fig. 7 so both beams can be combined and transported collinearlly on the axis of the target assembly. This allows both measurements to be made in the same spatial area. For the holography experiment an additional narrow band filter is used in front of the hologram to prevent any unwanted light from exposing the holographic film as shown in Fig. 7. Examples of the visible shadowgraphy are shown in Fig. 8. The figure shows three of twelve images obtained. The cross wires seen in the image are 12 micron wires. Mico-jets are seen to move radially inward.

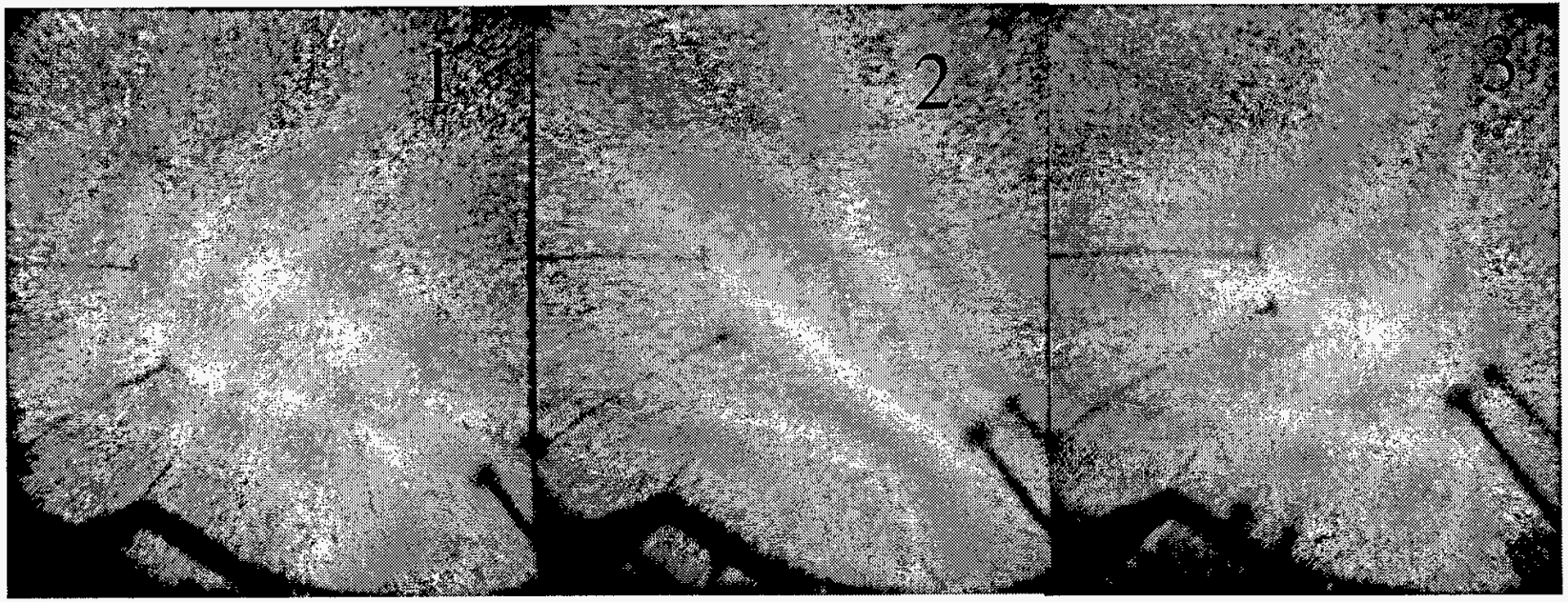

Figure 8: Shadowgraphs for three different times increasing from time from left to right. Micro-jets can be observed moving inward toward the center of the target assembly. Calibration cross hairs are seen which are 12 microns in diameter. 


\section{Conclusions}

The in-line Fraunhofer holography technique and visible shadowgraphy experimental techniques have been developed to be used in conjunction for ejecta measurements. Both measurements are made for the same spatial area. The holography provides high resolution three-dimensional data over about $1 \mathrm{~cm}^{3}$ for particles as small as 2 microns in diameter. The visible shadowgraphy provides spatially resolved images over a $170 \mathrm{~mm}^{2}$ area with an optical resolution of around 100 microns, but features as small as 10 microns in diameter are observed in the shadowgram. The shadowgraphy typically provides twelve images in time where each picture is gated at $50 \mathrm{~ns}$.

\section{Acknowledgements}

This work was supported by the United States Department of Energy

\footnotetext{
${ }^{1}$ P. Andriot, P. Chapron, V. Lambert, F. Olive, "Influence of Melting on Shocked Free Surface Behavior using Doppler Laser Interferometry and X-ray Densitometry", Shock Waves in Condensed Matter, Elsevier Science Publishers B.B., (1983).

2 J.R. Assay, "Material Ejection From Shock-Loaded Free Surfaces of Aluminum and Lead", Sandia Laboratories Report SAND 76-0542 (1976) (unpublished).

${ }^{3}$ P. Elias, P. Chapron, M. Mondot, "Experimental study of the slowing down of shock-induced matter ejection into argon gas". Shock Compression in Condensed Matter 1989, Elsevier Science Publishers B.B., (1990) 783-786.

${ }^{4}$ C. McMillan, R. Whipley, Proceedings 18th International Congress on High-Speed Photography and Photonics Xian, China (1988).

${ }^{5}$ J.L. Stokes, et. al., "Precision current measurements on Pegasus II using Faraday rotation", Proceedings of the 10th IEEE Pulsed Power Conference, Albuquerque, NM,1995, p. 378.

${ }^{6}$ L.R. Veeser, G.I. Chandler, and G.W. Day, "Fiber optic sensing of pulsed currents," Photonics: High Bandwidth Applications, James Chang, ed., (SPIE 648, Bellingham, WA, 1986), p. 197.

7 D. Platts, M. P. Hockaday, D. Beck, W. Coulter, R. C. Smith, "Compact Flash x-ray units," Proceedings of the 10th IEEE Pulsed Power Conference, Albuquerque, NM,1995, p. 892.

${ }^{8}$ D. S. Sorenson et al., "Particle Distribution Measurements Using In-Line Fraunhofer Holgoraphy," Proc. of the 22nd Int. Congress on High Speed Photography and Photonics ICHSPP'96, Santa Fe, NM, SPIE Vol. 2869, D.L. Paisley, A.M. Frank (eds.), p 206, 1996.

${ }^{9}$ D.S. Sorenson et al, "In-Line Particle Field Holography at Pegasus", Proceedings of the 10th IEEE Pulsed Power Confernece, Albuquerque, NM, 1995, p. 1024.

${ }^{10}$ Chandra S. Vikram, Particle Field Holography, Cambridge Studies in Modern Optics (1992).
} 
M97008so6

$\frac{L A-U R--97-2459}{\text { CONF-97061 }}$

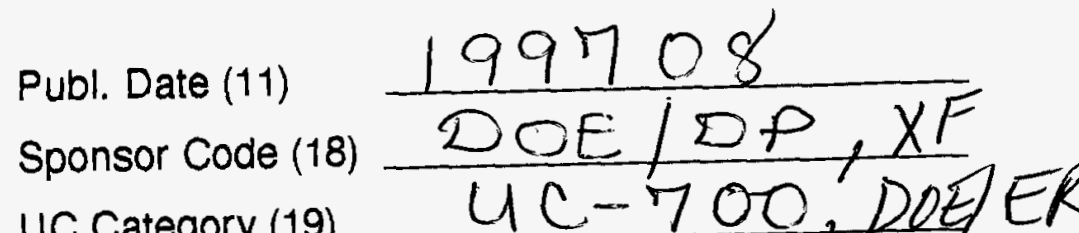

DOE 